\title{
Event Invitations in Privacy-Preserving DOSNs ${ }^{\star}$ Formalization and Protocol Design
}

\author{
Guillermo Rodríguez-Cano, Benjamin Greschbach, and Sonja Buchegger \\ KTH Royal Institute of Technology \\ School of Computer Science and Communication \\ Stockholm, Sweden \\ \{gurc, bgre, buc\}@csc.kth.se
}

The most common form of Online Social Networks (OSNs) are run in a logically centralized manner (although often physically distributed), where the provider operating the service acts as a communication channel between the individuals. Decentralization has been proposed to reduce the effect of these privacy threats by removing the central provider and its ability to collect and mine the data uploaded by the users as well as behavioral data.

One of the standard features of OSNs is the handling of event invitations and participation, i. e., a call for an assembly of individuals in the social graph for a particular purpose, e.g., a birthday celebration, demonstration, or meeting. There is usually metadata related to each event, such as date, location and a description. An implementation of this feature must provide security properties, e. g., that a user can verify that an invitation she received was actually sent by the organizer. Furthermore, it must support certain privacy settings. For example, an organizer could choose that only invited users learn how many other users were invited and that only after a user has committed to attend the event, she learns the identities of these other invited users.

Realizing this in a decentralized scenario is non-trivial because there is no Trusted Third Party (TTP) which all involved users can rely on. This is a problem especially for privacy properties where information shall only be disclosed to users with a certain status. In the example above, a neutral, trusted broker could keep the secret information (the identities of invited users) and disclose it only to users who committed to attend the event. This would guarantee fairness to both the organizer and the invited users. It becomes more challenging to implement this without a central TTP and still allowing different types of information about the event to be shared with different groups of users in a secure way.

In our proposal for a privacy-preserving decentralized implementation of the event invitation feature, as depicted in Figure 1, we divide the users of the Decentralized Online Social Network (DOSN) into the organizer of the event, the invitees, those who confirmed attending the event (attendees) and the remaining users. We assume basic functionality of popular OSNs to be available in a decentralized manner, such as user search or user messaging. We also assume

\footnotetext{
* A full paper on this work will be presented at the Privacy and Identity Management for the Future Internet in the Age of Globalisation - 9th IFIP WG 9.2, 9.5, 9.6/11.7, 11.4, 11.6 and Special Interest Group 9.2.2 International Summer School, Patras, Greece, September 7-12, 2014.
} 
that users are identified by a public key and the ability to verify the identity of other users via some sort of Public Key Infrastructure, which can be realized in a decentralized manner. Moreover, we rely on a distributed storage featuring access right management, e.g., that a certain storage object is only writeable by a specific user, and "append-only" storage objects, where new data can be appended, but existing data cannot be modified or removed without notice.

We describe and formally define two basic and five more complex security and privacy properties for the event invitations feature in DOSNs, such as invitee/attendee identity privacy (who learns the identities of the invitees/attendees), invitee/attendee count privacy (who learns the count of invitees/attendees), and attendee-only information reliability (availability of information exclusive to the attendees).

We also describe privacy enhancing tools, such as storage location indirection (to control not only who can decrypt an object but also who can see a ciphertext), controlled ciphertext inference (to allow a controlled information leak, e.g., about the size of an encrypted object to parties not able to decrypt the content) and a custom "commit-disclose protocol" to disclose a secret only to users who committed to attend an event. Using these tools together with standard cryptographic primitives, we discuss and propose a TTP-free architecture and decentralized protocols to implement the event invitation feature in a DOSN and analyze the usability and privacy implications. The suggested protocols cover all of our defined properties, considering 20 different parameter combinations for the tunable privacy properties.

The results can be applied in the context of Privacy-Preserving DOSNs, but might also be useful in other domains such as Working Environment and their corresponding collaborative-specific tools, i. e., groupware.

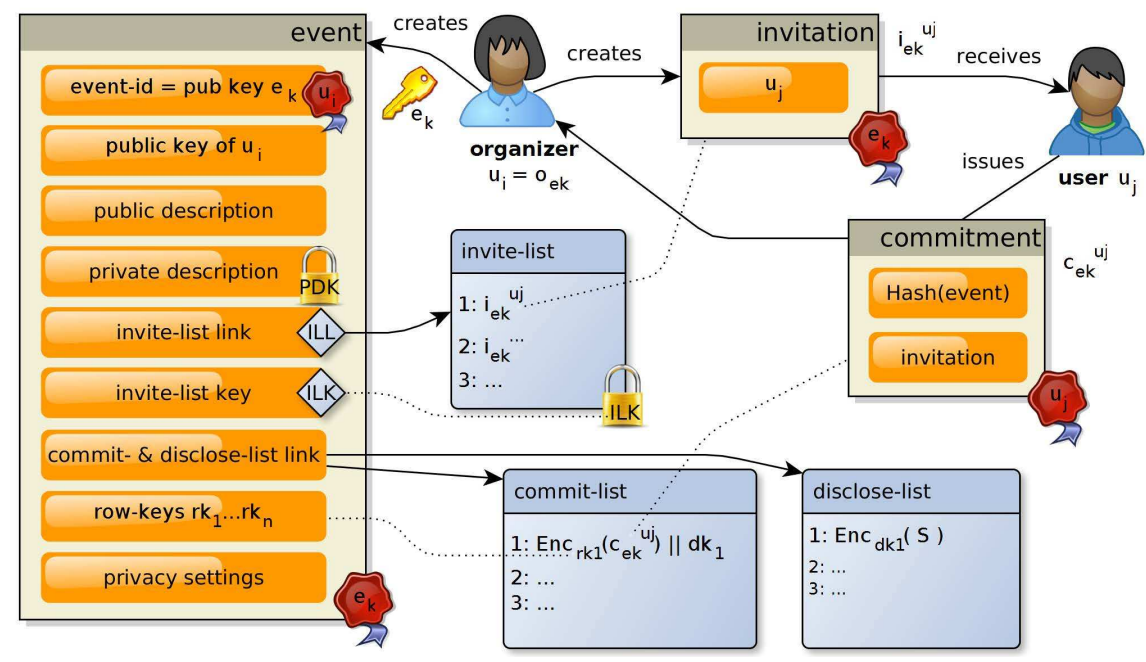

Fig. 1. Overview of system components and actions between stakeholders 